\title{
Approximate switching algorithms for trajectory surface hopping
}

\author{
E. Fabiano ${ }^{\mathrm{a}, *}$, G. Groenhof ${ }^{\mathrm{b}}$, W. Thiel ${ }^{\mathrm{a}}$ \\ ${ }^{a}$ Max-Planck-Institut für Kohlenforschung, Kaiser-Wilhelm-Platz 1, D-45470 Mülheim an der Ruhr, Germany \\ ${ }^{\mathrm{b}}$ Department of Theoretical and Computational Biophysics, Max-Planck-Institute for Biophysical Chemistry, Am Fassberg 11, D-37077 Gottingen, Germany
}

\section{A R T I C L E I N F O}

\section{Article history:}

Received 25 February 2008

Accepted 16 April 2008

Available online 20 April 2008

\section{Keywords:}

Surface hopping

Nonadiabatic dynamics

Potential-energy surfaces

Conical intersections

Nonadiabatic coupling terms

Semiempirical methods

\begin{abstract}
A B S T R A C T
Three approximate switching algorithms for trajectory surface hopping calculations are presented using simple models to describe the dependence of the hopping probability on the nonadiabatic coupling strength. The switching algorithms are applied to the calculation of the electronic deexcitation in ethylene, methaniminium ion and trans-azobenzene. Compared with the results from the standard fewest switching algorithm (FSA), the simplest approximation based on a local diabatic representation shows some qualitative failures and overestimates the decay times severely. The other two approximate switching algorithms incorporate stochastic features and reproduce the FSA results well, with a deviation of typically $20-30 \%$ in the computed decay times. They offer a simple and efficient description of the nonadiabatic dynamics of the investigated systems.
\end{abstract}

(c) 2008 Elsevier B.V. All rights reserved.

\section{Introduction}

Trajectory surface hopping (TSH) [1-12] is a general approach to study the dynamics of non-Born-Oppenheimer processes occurring in molecular systems. In this method the system is divided into a quantum and a classical part. The nonadiabatic dynamics is described by a swarm of classical trajectories with each trajectory evolving independently on a single electronic potential energy surface (PES) and the possibility of sudden instantaneous hops to different surfaces. However, there is not a generally accepted definition of how these transitions should be incorporated in the TSH method and the use of different hopping algorithms [1,3,5-7,1012] leads to slightly different TSH methods.

The most popular implementation of the TSH method is based on Tully's fewest switches algorithm (FSA) [5,6], which gives a good compromise between accuracy and computational efficiency. In the FSA the quantum amplitudes are propagated coherently along the trajectory and the transition probabilities are proportional to the variation of the quantum state populations. In addition, the number of transitions is required to be as small as possible. Although the FSA is conceptually very simple, its implementation requires the computation of nonadiabatic coupling vectors and the integration of quantum amplitudes at every point of the trajectory. Moreover, the exact transition probability is given by a complex expression that involves, in addition to nonadiabatic couplings, also the relative phases of the real and imaginary components of the quantum amplitudes. For these

\footnotetext{
* Corresponding author. Tel.: +492083062171.

E-mail address: fabiano@mpi-muelheim.mpg.de (E. Fabiano).
}

reasons the use of the FSA may be impractical for the study of large systems or for $\mathrm{QM} / \mathrm{MM}$ applications, where the explicit computation of nonadiabatic coupling vectors may no longer be feasible. In such cases it is desirable to use approximate switching methods that involve a numerical evaluation of the nonadiabatic couplings in terms of wavefunction overlap at successive time steps (see Section 2).

Approximate switching algorithms are commonly used in surface hopping studies on large systems [1,4,13-19], since they have a small computational cost and allow for a simple interpretation of the nonadiabatic dynamics. In particular, methods similar to the AS1 method (see below) have recently been applied in QM/MM studies of the photoactivation of the Photoactive Yellow Protein [16], in ab initio studies (CASSCF) of the photoisomerization of the retinal chromophore [17] and in QM/MM studies of the ultrafast deactivation of DNA base pairs [19]. However, to our knowledge, the intrinsic limitations of such approaches and their accuracy have not been investigated so far.

In this work we present a study on three approximate hopping methods. The first method (AS1) is based on a local diabatic hopping criterium and is a variant of a widely used approximate hopping algorithm $[16,17,19]$. We perform a full investigation of the capabilities and limitations of this approach. Furthermore, we introduce two new hopping algorithms (AS2, AS3) designed to correct the problems found in the AS1 approach. The three methods form a hierarchy of approximate switching algorithms that aim at reproducing the FSA results. The FSA is taken as reference, although it suffers from some well-known limitations $[10,12,20,21]$, because it is by far the most widely used method in molecular surface hopping calculations and usually yields 
good-quality results. The approximate switching algorithms are applied to three case studies, electronic deexcitation in ethylene, methaniminium ion and trans-azobenzene, and the results are compared with those obtained using the standard FSA approach. We find that the approximate switching algorithms introduced in this work, in particular the AS3 method, can reproduce the FSA results well and at the same time provide a simple and intuitive picture of nonadiabatic dynamics. In addition, since the computation of nonadiabatic coupling vectors and the integration of quantum amplitudes is avoided in these approximate methods, they are computationally efficient and easy to implement.

The article is organized as follows. In Section 2 the FSA is briefly reviewed and three approximate hopping algorithms are introduced. In Section 3 computational details are reported. In Section 4 the approximate hopping algorithms are applied to three test cases, and the results are compared with those from FSA. Finally, in Section 5 we present our conclusions.

\section{Theory}

\subsection{Fewest switches algorithm}

According to Tully's FSA $[5,6]$ the hopping probability is proportional to the variation of the quantum populations with the additional constraint that the number of hopping events is minimized. In the adiabatic representation the probability of hopping from the adiabatic surface $i$ to the adiabatic surface $j$ is

$P_{i j}=\max \left(q_{i j}, 0\right)$

with

$q_{i j}=\frac{2 \int_{t}^{t+\Delta t} \operatorname{Re}\left(c_{i}^{*}(\tau) c_{j}(\tau)\left[\overrightarrow{\vec{R}} \cdot \vec{d}_{i j}\right](\tau)\right) \mathrm{d} \tau}{c_{i}(t) c_{i}^{*}(t)}$

The complex-valued coefficients $c$ are the quantum amplitudes of the expansion of the total electronic wavefunction in terms of adiabatic states and are obtained from the solution of the time-dependent Schrödinger equation, $\vec{R}$ is the vector of nuclear velocities, and $\vec{d}_{i j}$ is the nonadiabatic coupling vector for the adiabatic states $i$ and $j$. The term $\left[\vec{R} \cdot \vec{d}_{i j}\right]$ is the nonadiabatic coupling between the adiabatic surfaces $i$ and $j$.

To determine whether a switch from state $i$ to state $j$ will occur at each time step a uniform random number $\xi \in[0,1]$ is selected and the hopping is performed if

$\sum_{k}^{j} P_{i k}<\xi<\sum_{k}^{j+1} P_{i j}$.

After the hopping the velocity in the direction of the nonadiabatic coupling vector $\vec{d}_{i j}$ is scaled in order to conserve the total energy of the system. If the scaling procedure is not sufficient to allow the conservation of total energy the hopping is rejected and the velocity components in the direction of the nonadiabatic coupling vector are reversed.

\subsection{Approximate switching algorithms}

In the FSA the hopping probability is proportional to the variation of the electronic populations. Since the changes in the electronic populations are driven by the magnitude of the nonadiabatic coupling, the hopping events occur only in regions of nonvanishing coupling and in particular in regions of high coupling, which are usually located close to the crossings (both allowed or avoided) of two adiabatic surfaces. Crossing regions are therefore particularly important for the description of surface hopping. This finding is also confirmed by the Landau-Zener formula for the hopping probability between two electronic states [22-24]

$P=\exp \left[-\frac{1}{4} \pi \zeta\right]$

The parameter $\zeta$ is the Massey parameter, defined as

$\zeta=\frac{\Delta E}{h D}$

where $\Delta E$ is the energy gap and $D$ is the nonadiabatic coupling between the two states. The hopping probability expressed by Eq. (4) is maximised when $\Delta E$ approaches zero and rapidly decays when $\Delta E$ increases. Moreover, the nonadiabatic coupling $D$ is largest when a diabatic crossing occurs. As a consequence the great majority of hopping events will occur in regions of very small energy gap and proceed through the diabatic crossing. Hence, in the development of approximate treatments, we first focus on such regions and use a local diabatic representation allowing the system to move on the diabatic surface. A switch between two adiabatic surfaces will thus occur if the diabatic surfaces cross.

A diabatic crossing can be characterized by the quantity

$s_{i j}=\left\langle\psi_{i}(t) \mid \psi_{j}(t+\Delta t)\right\rangle$

and the conditions

$s_{i i} \approx 0, \quad s_{j j} \approx 0$

$\left|s_{i j}\right| \approx 1, \quad\left|s_{j i}\right| \approx 1$,

where $|\psi\rangle$ denotes adiabatic states. We define a first approximate switching algorithm (AS1) as follows. When the energy gap between two adiabatic surfaces $i$ and $j$ is lower than a certain threshold $\Delta E$ we compute the average value of $\left|s_{i j}\right|$ and $\left|s_{j i}\right|$,

$\left|D_{i j}\right|=\left|\frac{\left\langle\psi_{i}(t) \mid \psi_{j}(t+\Delta t)\right\rangle-\left\langle\psi_{j}(t) \mid \psi_{i}(t+\Delta t)\right\rangle}{2}\right|$.

Comparison with Eq. (28) of Ref. [6] shows that

$D_{i j} \approx\left[\vec{R} \cdot \vec{d}_{i j}\right]_{(t+\Delta t / 2)} \Delta t$.

That is, $D_{i j}$ represents the nonadiabatic coupling over the time period $\Delta t$. The hopping $i \rightarrow j$ is performed if $\left|D_{i j}\right|$ exceeds a threshold $\Delta P$. In this work we used $\Delta E=30 \mathrm{kcal} / \mathrm{mol}$ and $\Delta P=0.5$. It turns out that conditions (7) are always satisfied whenever $\left|D_{i j}\right|>0.5$. After a hopping event a velocity adjustment is not strictly needed since in this approximation the system never leaves the diabatic surface. Tests indeed show that the use of a velocity adjustment procedure leaves the final results almost unchanged.

In the AS1 method weak nonadiabatic coupling regions are completely ignored because the hopping can occur only if a diabatic crossing occurs. However, when the FSA is employed, the weak coupling regions, if sufficiently extended, can play a significant role despite the fact that the hopping probability is very low at every single trajectory point. In fact, in the limit of an infinite region of small (or even infinitesimal) coupling the FSA yields a total hopping probability equal 1 , while AS1 yields 0 . This is a general deficiency of the AS1 method which can not be remedied by using a smaller value for $\Delta P$. On the contrary, in regions of high nonadiabatic coupling, too small values of the parameter $\Delta P$ may lead to repeated hopping and thus to loss of the independent trajectory description. Therefore it is advisable to relax the switching condition defining the AS1 method.

In our second approximate switching algorithm (AS2) the coupling $D_{i j}$ defined in Eq. (9) is computed when the energy gap is lower than a certain threshold $\Delta E$ (we use $\Delta E=30 \mathrm{kcal} / \mathrm{mol}$ ). A random number $\xi \in[0,1]$ is selected and the hopping $i \rightarrow j$ is performed if $\xi<\left|D_{i j}\right|$. After the hopping a velocity scaling is needed since, 
contrary to AS1, the system is no longer bounded to the diabatic surface. To avoid the computation of the nonadiabatic coupling vector the velocity scaling can be performed along the gradient difference vector. The effect of using the gradient difference in place of the nonadiabatic coupling vector is negligible $[25,26]$.

The AS2 method can be directly derived from the FSA by setting $P_{i j}=q_{i j}$ in Eq. (1) and assuming that in the time interval $\Delta t$ there is a uniform complete transfer of population from state $i$ to state $j$. In this case the quantum amplitudes (here supposed real for simplicity) in Eq. (2) are given by

$c_{i}(t)=1, \quad c_{i}(t+\Delta t / 2)=1 / \sqrt{2}, \quad c_{i}(t+\Delta t)=0$

$c_{j}(t)=0, \quad c_{j}(t+\Delta t / 2)=1 / \sqrt{2}, \quad c_{j}(t+\Delta t)=1$.

The integral in Eq. (2) can then be evaluated by the middle-point rule.

Disregarding approximations in the integral evaluation, the AS2 method and the FSA are equivalent if there is a uniform complete transfer of population from state $i$ to state $j$. This condition is very strong and not satisfied in general. In fact, in the FSA the changes in quantum amplitudes are usually rather small and the hopping probability is further reduced due to the different phases of real and imaginary parts of the quantum amplitudes. Moreover, according to Eq. (1), the FSA hopping probability is zero for those points in which the active state increases its quantum population (when $q_{i j}<0$ ). Therefore we can expect the AS2 method to overestimate the overall hopping probability since, in the FSA, there are some regions of non-negligible nonadiabatic coupling that do not contribute to the hopping process. Unfortunately, there are no simple arguments to estimate the change in the quantum amplitudes. A simple model can be used however to obtain information on the average hopping probability.

Consider a region of non-zero nonadiabatic coupling consisting of $N$ trajectory points. Suppose that the probability of hopping is $p$ at each point. The probability of moving across the entire region without hopping will be $(1-p)^{N}$ and the probability of hopping will be $P=1-(1-p)^{N}$. If $M$ points do not contribute to the hopping (i.e. they have $p=0$ ) the total hopping probability will be $P^{\prime}=1-(1-p)^{(N-M)}$. Equivalently the effective probability per point (including all $N$ points) is $p^{\prime}=1-(1-p)^{1-M / N}$.

In our third approximate switching algorithm (AS3) we extrapolate this simple model. As in the AS2 method, we suppose that the hopping probability is directly proportional to the magnitude of the nonadiabatic coupling $D_{i j}$. The trajectory is divided into small regions where the magnitude of the coupling is (almost) constant and the above reasoning is applied to every region. The coupling $D_{i j}$ defined in Eq. (9) is computed when the energy gap between two states is lower than a certain threshold $\Delta E$ (default: $\Delta E=30 \mathrm{kcal} / \mathrm{mol}$ ). The coupling is then scaled according to the formula

$D_{i j}^{\prime}=1-\left(1-D_{i j}\right)^{\alpha}$

where $0 \leqslant \alpha \leqslant 1$ is a parameter (see below). To decide if a hop should occur a random number $\xi \in[0,1]$ is selected and the hopping $i \rightarrow j$ is performed if $\xi<\left|D_{i j}^{\prime}\right|$. After a hopping event the velocity is adjusted along the gradient difference vector.

In principle the value of $\alpha$ should be different for every region of constant coupling and optimized according to the magnitude of $D_{i j}$. This is impractical in actual applications. Tests have shown, however, that the optimum $\alpha$ values vary only slightly in different regions and that $D_{i j}^{\prime}$ from Eq. (13) is rather insensitive to such small changes in $\alpha$. Therefore it is possible to employ an average value of $\alpha$ in Eq. (13) and treat it as a fixed parameter (we use $\alpha=0.5$ ).

\section{Computational details}

The TSH method has been applied to the description of the electronic deexcitation in ethylene, methaniminium ion and trans-azobenzene after vertical excitation from the ground state. Transition probabilities have been computed using the FSA and the three approximate switching algorithms described in Section 2.2.

The initial configurations for the TSH calculations were prepared by selecting a series of snapshots from a preliminary BornOppenheimer ground state MD run with the constraint that the vertical excitation energies of the initially populated state fall in the energy window $E_{0} \pm 0.15 \mathrm{eV}$, with $E_{0}$ being the vertical excitation energy at the ground state geometry optimized at the same level of computation. The same initial configurations were used to generate the swarms of trajectories for all the switching criteria examined.

Molecular orbitals were obtained from closed-shell OM2 $[27,28]$ calculations. Energies and gradients were determined from a GUGA configuration interaction (CI) treatment [29]. The nonadiabatic couplings were computed following the analytical procedure described in Refs. [30,31]. Other details of the calculations performed with the FSA are described in Ref. [32].

For ethylene a minimal $\mathrm{CI}$ treatment, with an active space comprising the HOMO and LUMO orbitals, was used to compute the lowest three electronic states. Initially the first excited state was populated and each trajectory was propagated for $200 \mathrm{fs}$ with a time step of $0.05 \mathrm{fs}$. The final average occupation of the states was obtained averaging over 80 trajectories.

In the case of the methaniminium ion the lowest three electronic states were computed by GUGA-CI using an active space consisting of four electrons and three orbitals (HOMO-1, HOMO, LUMO). At the beginning of the simulation the second excited state was populated. Each trajectory was then propagated for $150 \mathrm{fs}$ with a time step of $0.05 \mathrm{fs}$. The final results were obtained averaging over 80 trajectories.

The electronic deexcitation in azo-benzene was simulated considering only the ground and the first excited state $\left(n \rightarrow \pi^{*}\right.$ excitation). Electronic states were calculated by a GUGA-CI treatment with an active space consisting of eight electrons in seven orbitals (from HOMO-3 to LUMO+2). The excited state was initially populated and each trajectory was propagated for $1 \mathrm{ps}$ with a time step of $0.2 \mathrm{fs}$. The average occupation of the states was obtained by averaging over 40 trajectories.

All calculations were performed with the semiempirical MNDO package [33] including the recently developed TSH implementation [32]. The use of approximate switching algorithms reduced the overall computational time typically by $30-50 \%$ (compared with the FSA).

\section{Results}

\subsection{Ethylene}

Fig. 1 reports the average occupation of the lowest three states of ethylene computed using the FSA and the three approximate switching algorithms described in Section 2.2.

The FSA, AS2 and AS3 methods give a very similar description of the nonadiabatic dynamics of the ethylene. Upon excitation the molecule, initially in the $S_{1}$ state and with planar geometry, starts immediately a torsional motion around the $\mathrm{C}=\mathrm{C}$ bond followed by a pyramidalization of one of the two $\mathrm{CH}_{2}$ groups. During the torsional motion the molecule approaches the region of the twisted orthogonal conical intersection. In this region the $S_{1}$ and $S_{2}$ states experience a moderately high nonadiabatic coupling and hopping to the $S_{2}$ state is possible, although the system usually rapidly 


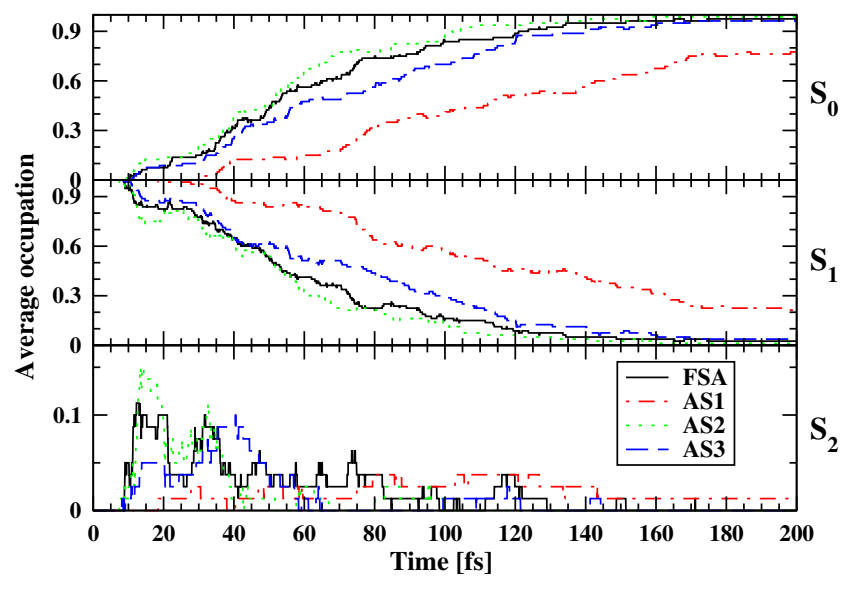

Fig. 1. Average occupation of the three lowest states of ethylene computed using the fewest switches algorithm (FSA) and the AS1, AS2 and AS3 switching methods described in Section 2.2.

decays back to the $S_{1}$ state. Increasing pyramidalization brings the molecule towards the twisted-pyramidalized conical intersection. In the twisted-pyramidalized configuration the $S_{1}$ and $S_{0}$ states are strongly coupled and hopping from the $S_{1}$ state to the $S_{0}$ state is highly probable. In a small number of trajectories the $S_{1} \rightarrow S_{0}$ deexcitation follows a different path and proceeds through a hydrogen migration mechanism (ethylidene conical intersection). Similar results are found also in other TSH studies [34-37] and in quantum wavepacket calculations [38,39].

In Table 1 the decay time $\tau$ of the $S_{1}$ state, calculated using a single exponential decay model, is reported for all the hopping methods. Compared with the FSA the AS2 method yields a slightly smaller decay time and the AS3 method a slightly larger one. Both methods give results close to those obtained with the FSA.

Application of the AS1 method leads to an underestimation of the hopping probability. Due to the stiff hopping criterion of the AS1 method and the fact that the nonadiabatic coupling around the twisted orthogonal conical intersection is not very high, the molecule needs to reach a configuration very close to the conical intersection before transitions between the $S_{1}$ and the $S_{2}$ state are possible. Therefore the probability of hopping from state $S_{1}$ to state $S_{2}$ is very low. For similar reasons if the molecule switches to the $S_{2}$ state it can not easily decay back to the $S_{1}$ state (as usually happens in the FSA description) and the final $S_{2}$ average population computed with the AS1 method is higher than that computed with the FSA. Similar considerations hold for the $S_{1} \rightarrow S_{0}$ hopping but in this case the two states are strongly coupled in the region of the twisted pyramidalized conical intersection and the AS1 method works better. The increase in the $\mathrm{S}_{0}$ occupation is slower than that computed with the FSA but follows a similar trend. Part of the difference in the predicted occupations is also due to the fact that the $\mathrm{S}_{0}$ state starts to be populated at $t \approx 20$ fs in the AS1 case, and already at $t \approx 9$ fs in the FSA case because the molecule must be closer to the conical intersection before a hopping is allowed in the AS1 method.

Table 1

Decay times in fs for the excited states of ethylene, methaniminium ion and transazobenzene calculated with the fewest switches algorithm (FSA) and the three approximate switches algorithm described in Section 2.2

\begin{tabular}{llrrrr}
\hline System & State & FSA & AS1 & AS2 & AS3 \\
\hline Ethylene & $\mathrm{S}_{1}$ & 64 & 164 & 57 & 80 \\
Methaniminium & $\mathrm{S}_{2}$ & 15 & 224 & 10 & 14 \\
\multirow{3}{*}{ Azobenzene } & $\mathrm{S}_{1}$ & 97 & 215 & 63 & 93 \\
& $\mathrm{~S}_{1}$ & 664 & 2993 & 265 & 394 \\
\hline
\end{tabular}

\subsection{Methaniminium ion}

The average occupation of the lowest three states of the methaniminium ion computed using the FSA and the three approximate switching algorithms described in Section 2.2 is reported in Fig. 2.

The FSA predicts a fast $S_{2} \rightarrow S_{1}$ deexcitation through the planar $\mathrm{S}_{2} / \mathrm{S}_{1}$ conical intersection. After this first hopping two pathways are possible for the $S_{1} \rightarrow S_{0}$ deexcitation. For the majority of trajectories the $S_{1} \rightarrow S_{0}$ hopping occurs in the region of the twisted conical intersection, because of the high nonadiabatic coupling characterizing this configuration. In about $30 \%$ of the trajectories the hopping occurs instead in a configuration characterized by a very large $\mathrm{C}-\mathrm{N}$ bond distance and bipyramidalization. This mechanism is often associated with a very early $S_{2} \rightarrow S_{1}$ hopping (in agreement with the dynamical interpretation suggested in Ref. [40]) and offers a faster decay path with respect to the first one. However, the occurrence of a hopping event in the stretched-bipyramidalized configuration is disfavored since in this configuration the nonadiabatic coupling is not as high as in the region of the twisted conical intersection and since the quantum population of the ground state grows very slowly along the stretching-bipyramidalization reaction coordinate. Similar findings are reported in previous TSH investigations [40-42].

The AS1 method fails in providing a correct description of the $S_{2} \rightarrow S_{1}$ hopping. The nonadiabatic coupling between the $S_{2}$ and the $S_{1}$ state is never particularly high, but of moderate strength over a large part of configurational space. In this situation the FSA hopping probability is high but the coupling is rarely strong enough to allow for hopping at the AS1 level. As a consequence about one half of the trajectories evolve only on the $S_{2}$ adiabatic surface at the AS1 level and in any case the $S_{2}$ decay time is strongly overestimated (see Table 1 ). Thus, the average occupation of the $S_{1}$ state grows slowly and its deexcitation dynamics at the AS1 level can be hardly compared with that predicted by the FSA. For the AS1 method, the twisted conical intersection pathway is almost the only channel for the $\mathrm{S}_{1} \rightarrow \mathrm{S}_{0}$ deexcitation and almost no hopping occurs along the stretching-bipyramidalization pathway due to its reduced nonadiabatic coupling.

The description of the $S_{2} \rightarrow S_{1}$ hopping is strongly improved when using the AS2 method although the $S_{2}$ decay time is slightly underestimated. The $\mathrm{S}_{1} \rightarrow \mathrm{S}_{0}$ hopping events are evenly distributed among the two decay paths described for the FSA case. The number of trajectories evolving through the stretching-bipyramidalized path is overestimated due to the fact that the AS2 method makes hopping too easy in these configurations and neglects the role of

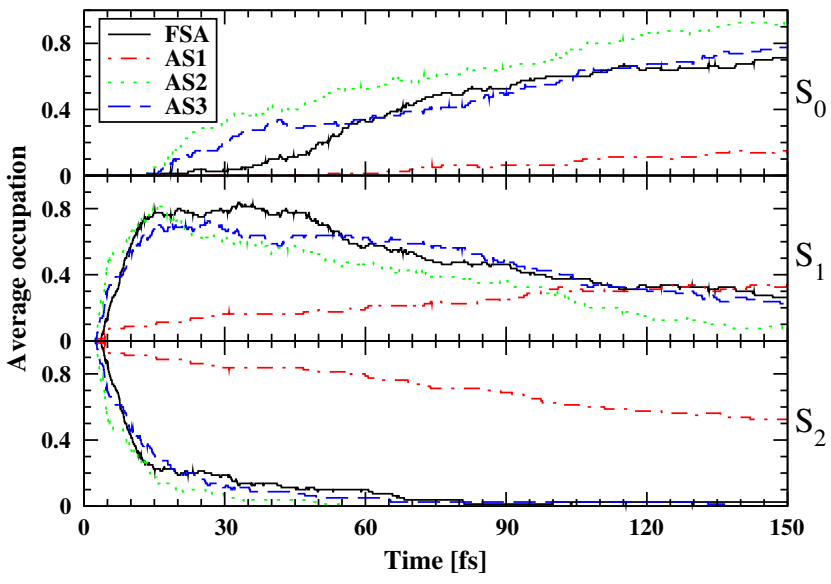

Fig. 2. Average occupation of the three lowest states of methaniminium ion computed using the fewest switches algorithm (FSA) and the AS1, AS2 and AS3 switching methods described in Section 2.2. 
the population/depopulation of quantum states. As a result of having almost half of the trajectories in the fast stretching-bipyramidalization deexcitation path, the $S_{1}$ decay time is computed to be $63 \mathrm{fs}$, smaller than at the FSA level. Note however that a decay time similar to that computed with the AS2 method is found in a recent ab initio study [42] where a similar ratio between the two decay paths is observed.

The AS3 method yields results very similar to the FSA. In particular it avoids the problems of the AS2 method in the description of the $S_{1} \rightarrow S_{0}$ hopping and correctly assigns the relative number of trajectories to each of the deexcitation paths. A small overestimation of the $\mathrm{S}_{1} \rightarrow \mathrm{S}_{0}$ hopping probability is still observed between 20 and $50 \mathrm{fs}$. In this time interval the small quantum occupation of the $\mathrm{S}_{0}$ state makes the FSA hopping probability rather low, but this effect is ignored by the AS3 method.

\subsection{Trans-azobenzene}

The average occupation of the first excited state $\left(n \rightarrow \pi^{*}\right.$ transition) of the trans-azobenzene molecule after vertical excitation from the ground state has been computed using the FSA and the three approximate switching algorithms described in Section 2.2. The results are shown in Fig. 3.

After the excitation there is a slow torsion around the $\mathrm{N}=\mathrm{N}$ double bond accompanied by fast oscillations of the NNC angles. During this motion the molecule is repeatedly brought into the proximity of the $S_{0} / S_{1}$ conical intersection, each time with slightly different values of the CNNC dihedral angle and the NNC angles. The magnitude of the nonadiabatic coupling depends on how closely the conical intersection is approached. At the beginning, for relatively small values of the torsional angle, there is only a small coupling. The magnitude of the nonadiabatic coupling increases successively as the torsional angle and the NCC angles move towards $\sim 90^{\circ}$ and $\sim 120^{\circ}$, respectively. Finally, the nonadiabatic coupling is again reduced when the NNC angles slowly drift to larger values. This means that the decay of the excited state occurs only during a limited time period (lasting $\sim 400 \mathrm{fs}$ ) which is preceded and followed by periods where the average occupation of the excited state is almost constant. Similar observations are described in previous TSH studies [43-45] and wavepacket dynamics investigations [44].

The behavior outlined above is captured rather well by the AS2 and AS3 methods although both predict the beginning of excited state decay too early. This difference can be rationalized consider-

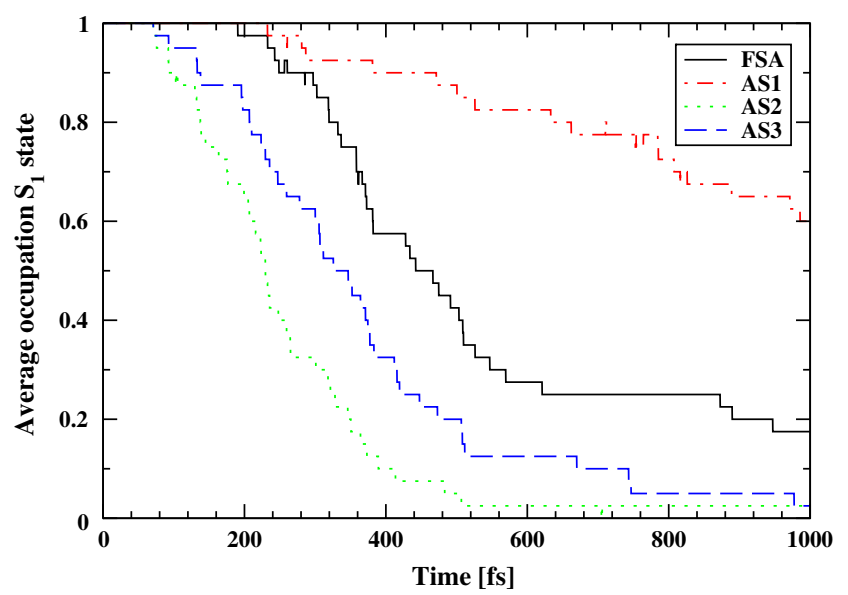

Fig. 3. Average occupation of the excited state of the trans-azobenzene molecule computed using the fewest switches algorithm (FSA) and the AS1, AS2 and AS3 switching methods described in section 2.2. ing that the FSA hopping probability depends not only on the magnitude of the nonadiabatic coupling, but also on the absolute value of the quantum population of the ground state. Before the ground state quantum population can grow to a significant value the molecule must cross a coupling region several times. This shifts the beginning of the excited state decay to a later time in the FSA description. The AS2 method predicts a decay time $\tau=265$ fs which is too short compared to $\tau=664$ fs from the FSA. The use of the AS3 method leads to some improvement. The computed value $\tau=393 \mathrm{fs}$ is however still too small with respect to the FSA result. The difference between the two results comes mainly from the fact that the FSA decay starts at a later time than the AS3 decay, and possibly also from the use of an $\alpha$ parameter in the AS3 model that has not been optimized.

In the AS1 method only configurations very close to the $S_{0} / S_{1}$ conical intersection yield a sufficiently high coupling to allow the hopping. As a result a very slow decay of the excited state is predicted. The decay time is almost three times larger than that predicted with the FSA, and after 1 ps only about $40 \%$ of the trajectories are propagating on the ground state PES.

\section{Conclusions}

The fewest switches algorithm is the standard approach for the computation of the hopping probability in TSH calculations. The FSA is based on the minimization of the number of transitions needed to maintain the self-consistency between the quantum and the classical populations. In its practical implementation the FSA requires the knowledge of the nonadiabatic couplings and the coherent integration of the quantum amplitudes along the entire trajectory. This information is then used in Eqs. (1) and (2) to compute the hopping probability. These requirements can not be easily fullfilled in applications on large systems, if one insists on an explicit evaluation of the nonadiabatic coupling vectors, and therefore the FSA is rarely applied in these studies. This motivates attempts to describe the relationship between the magnitude of the nonadiabatic coupling and the hopping probability by simpler models. The corresponding approximate algorithms should offer a simple picture of the nonadiabatic dynamics and, at the same time, reproduce the hopping probabilities with good accuracy.

In this work we introduced three approximate switching algorithms for TSH calculations. The performance of the three approximate switching algorithms against the FSA was tested by applying them to the calculation of the electronic deexcitation in ethylene, methaniminium ion and trans-azobenzene.

The first approximate method (AS1) is based on a local diabatic approximation for the interaction region. This method can qualitatively capture the main features of the hopping process but is too simple to yield accurate quantitative predictions. The hopping probability is generally underestimated and the decay time of excited states is on average five times larger than that computed at the FSA level.

The main weakness of the AS1 method is that it does not incorporate any contribution from regions with weak nonadiabatic coupling. This limitation is overcome in the AS2 method by using a stochastic procedure to determine whether a hopping event should occur. The AS2 methods performs much better than the AS1 method and predicts the decay times typically within $30 \%$ of the FSA values.

The AS2 method tends to overestimate hopping probabilities because it does not consider the effect of quantum depopulation on the hopping probability. This is corrected in the AS3 method with the introduction of an effective hopping probability, depending on a parameter $\alpha$. The parameter $\alpha$ was not optimized in this study, but fixed to the value of 0.5 . With this choice the AS3 
method improves on the performance of the AS2 method and yields decay times that deviate on the average by only $19 \%$ from the FSA values.

The presence of a parameter in the AS3 method offers the opportunity to optimize it against reference targets other than the FSA. It would be particularly interesting to find out whether the AS3 method can partially correct the well known drawbacks of the FSA $[10,12,20,21]$ by calibrating it against the results of quantum wavepacket calculations [46-52]. This is a topic for future studies.

\section{Acknowledgement}

This work was supported by the Deutsche Forschungsgemeinschaft (SFB 663).

\section{References}

[1] J.C. Tully, R.K. Preston, J. Chem. Phys. 55 (1971) 562.

[2] W.H. Miller, T.F. George, J. Chem. Phys. 56 (1972) 5637.

[3] J.R. Stine, J.T. Muckerman, J. Chem. Phys. 65 (1976) 3975.

[4] G. Parlant, E.A. Gislason, J. Chem. Phys. 91 (1989) 4416.

[5] J.C. Tully, J. Chem. Phys. 93 (1990) 1061.

[6] S. Hammes-Schiffer, J.C. Tully, J. Chem. Phys. 101 (1994) 4657.

[7] D.F. Coker, L. Xiao, J. Chem. Phys. 102 (1995) 496.

[8] J.C. Tully, Faraday Discuss. 110 (1998) 407.

[9] D.S. Sholl, J.C. Tully, J. Chem. Phys. 109 (1998) 7702

[10] J.-Y. Fang, S. Hammes-Schiffer, J. Phys. Chem. A 103 (1999) 9399.

[11] A. Bastida, C. Cruz, J. Zúñiga, A. Requena, J. Chem. Phys. 119 (2003) 6489.

[12] G. Granucci, M. Persico, J. Chem. Phys. 126 (2007) 134114.

[13] G.A. Jones, B.K. Carpenter, M.N. Paddon-Row, J. Am. Chem. Soc. 120 (1998) 5499.

[14] E.E. Nikitin, Ann. Rev. Phys. Chem. 50 (1999) 1.

[15] M. Meuwly, O.M. Becker, R. Stote, M. Karplus, Biophys. Chem. 98 (2002) 183.

[16] G. Groenhof, M. Bouxin-Cademartory, B. Hess, S.P. de Visser, H.J.C. Berendsen, M. Olivucci, A.E. Mark, M.A. Robb, J. Am. Chem. Soc. 126 (2004) 4228.

[17] O. Weingart, I. Schapiro, V. Buss, J. Mol. Model. 12 (2006) 713.

[18] M.J. Bearpark, M. Boggio-Pasqua, M.A. Robb, F. Ogliaro, Theor. Chem. Acc. 116 (2006) 670.
[19] G. Groenhof, L.V. Schäfer, M. Boggio-Pasqua, M. Goette, H. Grubmüller, M.A. Robb, J. Am. Chem. Soc. 129 (2007) 6812.

[20] U. Müller, G. Stock, J. Chem. Phys. 107 (1997) 6230.

[21] A.W. Jasper, S.N. Stechmann, D.G. Truhlar, J. Chem. Phys. 116 (2002) 5424.

[22] L.D. Landau, Phys. Z. Sowjetunion 2 (1932) 46.

[23] C. Zener, Proc. R. Soc. London, A 137 (1932) 692

[24] C. Wittig, J. Phys. Chem. B 109 (2005) 8428.

[25] M.S. Topaler, T.C. Allison, D.W. Schwenke, D.G. Truhlar, J. Phys. Chem. A 102 (1998) 1666.

[26] M.D. Hack, D.G. Truhlar, J. Phys. Chem. A. 104 (2000) 7917.

[27] W. Weber, Ph.D. Thesis, Universität Zürich, Switzerland, 1996.

[28] W. Weber, W. Thiel, Theor. Chem. Acc. 103 (2000) 495.

[29] A. Koslowski, M.E. Beck, W. Thiel, J. Comput. Chem. 24 (2003) 714.

[30] B.H. Lengsfield III, D.R. Yarkony, Adv. Chem. Phys. 82 (1992) 1.

[31] T.W. Keal, A. Koslowski, W. Thiel, Theor. Chem. Acc. 118 (2007) 837.

[32] E. Fabiano, T.W. Keal, W. Thiel, Chem. Phys. (2008), doi:10.1016/ j.chemphys.2008.01.044.

[33] W. Thiel, MNDO program version 6.1, Mülheim, 2007.

[34] G. Granucci, M. Persico, A. Toniolo, J. Chem. Phys. 114 (2001) 10608

[35] M. Barbatti, J. Paier, H. Lischka, J. Chem. Phys. 121 (2004) 11614

[36] M. Barbatti, G. Granucci, M. Persico, H. Lischka, Chem. Phys. Lett. 401 (2005) 276

37] M. Barbatti, M. Ruckenbauer, H. Lischka, J. Chem. Phys. 122 (2005) 174307.

[38] M. Ben-Nun, T.J. Martinez, Chem. Phys. Lett. 298 (1998) 57.

[39] J. Quenneville, M. Ben-Nun, T.J. Martinez, J. Photochem. Photobiol. A 144 (2001) 229.

[40] M. Barbatti, A.J.A. Aquino, H. Lischka, Mol. Phys. 104 (2006) 1053.

[41] E. Tapavicza, I. Tavernelli, U. Röthlisberger, Phys. Rev. Lett. 98 (2007) 023001

[42] M. Barbatti, G. Granucci, M. Persico, M. Ruckenbauer, M. Vazdar, M. EckertMaksić, H. Lischka, J. Photochem. Photobiol. A 190 (2007) 228.

[43] C. Ciminelli, G. Granucci, M. Persico, Chem. Eur. J. 10 (2004) 2327.

[44] A. Toniolo, C. Ciminelli, M. Persico, T.J. Martinez, J. Chem. Phys. 123 (2005) 234308.

[45] G. Granucci, M. Persico, Theor. Chem. Acc. 117 (2007) 1131.

[46] E.J. Heller, J.R. Reimers, in: J. Broekchove, L. Lathouwers, P. Van Leuwen (Eds.), Dynamics of Wave Packets in Molecular and Nuclear Physics, Springer, Berlin, Heidelberg, 1986, p. 79.

[47] B.M. Garraway, K.-A. Suominen, Rep. Prog. Phys. 58 (1995) 365.

[48] J. Morelli, S. Hammes-Schiffer, Chem. Phys. Lett. 269 (1997) 161.

[49] H. Nagao, K. Kodama, Y. Shigeta, K. Nishikawa, H. Kawabe, M. Nakano, K. Yamaguchi, Int. J. Quantum Chem. 60 (1998) 1261.

[50] M. Ben-Nun, J. Quenneville, T.J. Martinez, J. Phys. Chem. 104 (2000) 5161.

[51] M. Ben-Nun, T.J. Martinez, Adv. Chem. Phys. 121 (2002) 439.

[52] G.A. Worth, P. Hunt, M.A. Robb, J. Phys. Chem. A 107 (2003) 621. 